\title{
Determinantes de la participación femenina en el mercado de trabajo en la Galicia rural y urbana de 1924
}

\author{
Luisa María Muñoz Abeledo, María Salomé Taboada \\ y Rosa María Verdugo
}

PALABRAS CLAVE: actividad femenina, demanda y oferta de trabajo, empleo, ingresos familiares.

CÓdIGOS JEL: J20, J21, J23, D19.

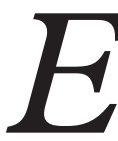

ste artículo ofrece nuevos datos sobre la tasa de actividad femenina mediante

el análisis de los determinantes de participación de las mujeres en el mercado

laboral de Galicia en 1924. En esta investigación se eligieron cinco municipios con modelos económicos diferentes: dos ciudades y tres pueblos. Las ciudades son A Coruña, puerto comercial, industrial y de servicios, y Ourense, una capital del interior de la región. Los municipios rurales analizados son: Bueu, que modeliza la industrialización del litoral, centrado en la pesca y su transformación; Padrón, que combina agricultura, textil y curtidos; y finalmente, Nigrán, eminentemente agrario. Combinando datos demográficos (el padrón de 1924) con otras fuentes, este artículo corrige el subregistro de la actividad femenina en la agricultura y en la industria de transformación de pescado, al obtener tasas que superan el 50\% en los municipios rurales y el 30\% en los urbanos. Además, el artículo analiza el funcionamiento de los mercados de trabajo del mundo rural y urbano desde una perspectiva de género, identificando las principales ocupaciones masculinas y femeninas. Por último, explora si se cumple el modelo del hombre como principal sustentador de pan en los hogares de la región en los años veinte del siglo $\mathrm{XX}$. 


\title{
Female labour participation rates in rural and urban Galicia in 1924
}

\author{
KEYWORDS: female labour activity, labour supply and demand, \\ employment, family income.
}

JEL CODES: J20, J21, J23, D19.

his article provides new data on the female labour activity rate, obtained by
analysing the determinants of female participation in rural and urban labour
markets in Galicia in 1924. We selected five municipalities (two cities and three towns) to represent different economic models. The two larger hubs are A Coruña, a city with industry, services and a commercial port; and Ourense, a provincial capital in the interior of the region. The smaller, more rural municipalities analysed are Bueu, a good example of the region's industrialization model focused on fishing, fish processing; Padrón, which combines agriculture, textiles, and tanning sectors; and Nigrán, which is eminently agrarian. By combining demographic data (Nominative Population Census of 1924) with other sources, this article corrects the female activity rate in agriculture and the fishprocessing industry. The revised female labour participation rates are higher, surpassing 50\% in rural municipalities and 30\% in cities. We also analyse rural and urban labour markets from a gender perspective, identifying the main male and female occupations. Finally, this research explores the accuracy of the predominance of the "male breadwinner" model in this region in the 1920s.

Recibido: 2018-06-02 - Revisado: 2019-02-25 - Aceptado: 2019-02-25

Luisa María Muñoz Abeledo [orcid.org/0000-0002-7970-3045] es titular de universidad de Historia Contemporánea en la Universidad de Santiago de Compostela. Dirección para correspondencia: Historia Contemporánea, Facultad de Geografía e Historia, Universidad de Santiago de Compostela, Praza da Universidade,n. ${ }^{\circ}$ 1, 15782 Santiago de Compostela (España).C.e.:luisamaria.munoz@usc.es

María Salomé Taboada Mella [orcid.org/0000-0003-4491-2645] es titular de escuela universitaria de Economía Financiera y Contabilidad en la Universidad de Santiago de Compostela. Dirección para correspondencia: Departamento de Economía Financiera y Contabilidad, Facultad de Ciencias Económicas y Empresariales, Universidad de Santiago de Compostela, Campus Norte, 15782 Santiago de Compostela (España).C.e.: msalome.taboada@usc.es

Rosa María Verdugo Matés [orcid.org/0000-0001-9842-3391] es profesora contratada doctora de Economía Aplicada en la Universidad de Santiago de Compostela. Dirección para correspondencia: Departamento de Economía Aplicada, Facultad de Ciencias Económicas y Empresariales, Universidad de Santiago de Compostela, Campus Norte, 15782 Santiago de Compostela (España).C.e.: rosa.verdugo@usc.es 


\section{INTRODUCCIÓN}

La literatura española sobre la participación femenina en el mercado de trabajo desde una perspectiva histórica ha sido prolífica en las últimas décadas. Estudios recientes recogen evidencias cuantitativas y cualitativas sobre el trabajo de las mujeres en la España preindustrial, tanto en la agricultura (Jover, Pujadas \& Suau, 2017; Ortega, 2015) como en las manufacturas rurales (Martínez López \& Martínez Martín, 2003; Sarasúa, 2000, 2013, 2019; Garrido, 2016; Hernández García \& Cubero, 2017) y también en los diferentes modelos de industrialización (Humphries \& Sarasúa, 2012; Muñoz Abeledo, 2012a, 2012b; Muñoz Abeledo, Taboada \& Verdugo, 2015; Borderías, 2012, 2013a, 2013b; Borderías \& Ferrer, 2015, 2017; Pérez-Fuentes, 1995; Campos, 2014; Borrás, 2012, 2013; Martínez Soto, 2013). Estas investigaciones han corregido el subregistro y recalculado la tasa de actividad para diferentes regiones e industrias en la España contemporánea (Gálvez Muñoz, 1997; Arbaiza, 2000, 2002; Camps, 1995, 1999; Borderías, 2010a, 2010b, 2012, 2013a, 2013b; Borderías \& Ferrer, 2015; Pérez-Fuentes, 2003, 2013; Pérez-Fuentes \& Pareja, 2011; Martínez Martín \& Moya, 2011; Muñoz Abeledo, 2012a, 2012b, 2013; Muñoz Abeledo, Taboada \& Verdugo, 2015; Campos, 2014; Garrido, 2016; Borrás, 2012, 2013; Martínez Soto, 2013). Este subregistro de la actividad femenina también se dio en otros países europeos (los casos más analizados son los de Reino Unido, Francia y Países Bajos), donde resultó especialmente elevado cuando las mujeres se dedicaban a la agricultura (Folbre, 1995; Grantham, 2012; Grantham \& Grimard, 2010; Higgs, 1987, 1995, 2005; Horrell \& Humphries, 1995, 1997; Humphries \& Sarasúa, 2012; Janssens, 1997; Nederveen Meerkerk, 2010; Verdon, 2002).

Los objetivos de este artículo, todos ellos centrados en la Galicia de la década de 1920, son cuatro. En primer lugar, corregir el subregistro de la actividad femenina oculta en los padrones y censos de población. En publicaciones anteriores se ha calculado esta tasa para la Galicia de mediados del siglo xIx (Muñoz Abeledo, Taboada \& Verdugo, 2015). A pesar de que en Galicia no existen, como en el caso catalán, los censos obreros para 1920 (Borderías, 2012, 2013a), se ha corregido el subregistro del padrón de 1924, para algunos sectores representativos del proceso de industrialización regional, combinando datos demográficos con datos de empresa; y, para la agricultura, corrigiendo el padrón tal y como se expone en el apartado metodológico. En segundo lugar, se explica el funcionamiento de los mercados de trabajo del mundo rural y urbano desde una perspectiva de género, identificando las principales ocupaciones masculinas y femeninas. En tercer lugar, se exploran los condicionantes de la actividad femenina, tanto de oferta (número de activos e inactivos de la unidad familiar, hijos pequeños e hijos que trabajan y salario del marido para las casadas) como de demanda (empresas que empleaban mano de obra femenina). Por último, se explora de forma tentativa si existía el modelo del ganador de pan 
en los años veinte del siglo pasado, y para ello se utiliza también el padrón de 1924, porque es el único con información salarial (Borderías \& Muñoz Abeledo, 2018). A pesar de que en él no fueron registrados los ingresos para todos los hogares (declaraban salario generalmente los cabezas de casa y, en menor medida, esposas, hijos y parientes), esta fuente permite establecer hipótesis sobre si la actividad de las mujeres dependía del salario de los maridos, habida cuenta de que, precisamente, la década de los veinte ha sido considerada por la historiografía como la de consolidación del modelo de ganador de pan debido al incremento de los salarios de los obreros varones que tuvo lugar durante y tras la Primera Guerra Mundial (Camps, 1991, 1995; Ballesteros, 2002; Lana, 2007; Maluquer de Motes, 2005, 2006, 2013; Escudero, 2002, 2003; Silvestre, 2005; Pérez Castroviejo, 1992, 2006; Llonch, 2004; Escudero \& Pérez Castroviejo, 2010; Vilar, 2014).

\section{FUENTES Y METODOLOGÍA}

En este artículo se calcula la tasa de actividad femenina (en adelante, TAF) de cinco municipios de la Galicia del primer cuarto del siglo xx que ejemplifican modelos socioeconómicos distintos: dos urbanos (A Coruña y Ourense) y tres rurales (Bueu, Padrón y Nigrán). Las dos ciudades seleccionadas eran, a comienzos del siglo $\mathrm{xx}$, centros administrativos, de comercio y de servicios, pero con importantes diferencias entre ellas: A Coruña era la ciudad más poblada de Galicia (62.022 habitantes, según el censo de la población de España en 1920) y contaba con una base industrial diversificada y dinámica, mientras que Ourense era una pequeña capital de provincia (17.581 habitantes) con poca industria y mucha agricultura en los barrios periféricos. Los municipios rurales elegidos muestran, a un nivel micro, algunos de los sectores más típicos de la estructura económica de la región, dominada por la agricultura, la pesca y su transformación: Nigrán era un municipio fundamentalmente agrario situado en la ría de Vigo y contaba con 6.918 habitantes; Padrón, un pueblo de A Coruña de 7.148 habitantes, combinaba la agricultura con el textil y la industria de curtidos (Freire, 2008); por último, Bueu, situado en la ría de Pontevedra y con 8.411 habitantes, era un buen ejemplo de los pueblos pesqueros con industria conservera (Muñoz Abeledo, 2010).

En esta investigación se recurre a dos tipos de fuentes: los padrones y los censos de población de la década de 1920. El censo de 1920 clasifica a los habitantes según sexo, edad, estado civil, instrucción elemental y profesión para cada una de las provincias y capitales. En lo que respecta a la ocupación, esta fuente clasifica a los trabajadores en 80 grupos de industrias y profesiones: agricultura, industria y comercio (del grupo 1 al 47), fuerza pública (48-50), administración (51-52), culto y clero (53-56), profesiones liberales (57-65), personas que viven de sus rentas (66-67), retirados o pensionistas del Es- 
tado y de otras administraciones públicas y privadas (68), sirvientes domésticos (69), individuos momentáneamente sin ocupación (70-79). Para conocer el número de personas ocupadas a partir de este censo, hemos considerado todos los grupos con la excepción del 68 y del 70 al $80^{1}$.

Esta fuente presenta varias limitaciones para realizar el cálculo de la tasa de actividad: en primer lugar, la división de la población según profesión solo se realiza para las provincias y sus capitales, lo que imposibilita el análisis a nivel municipal. En segundo lugar, para las capitales se proporciona información por grupos de edad muy amplios (menores de 21 años, entre 21 y 60, y mayores de 60), lo que dificulta el cálculo detallado de la actividad a lo largo del ciclo vital. En tercer lugar, el censo presenta un elevado subregistro del trabajo de la mujer, ya que se anota su ocupación solo cuando es cabeza de familia, viuda o casada con marido ausente, mientras que la profesión del hombre figura siempre. Esta forma de operar obedecía a la ideología imperante en la España de la época que potenciaba el discurso de la domesticidad, según el cual las mujeres tenían que dedicarse a las tareas del hogar y al cuidado de la familia, y dejar para los varones el trabajo fuera de casa (Arbaiza, 2000, 2002, 2003; Borderías, 1993, 2003; Camps, 1995; Gálvez Muñoz, 2000; Muñoz Abeledo, 2010; Pérez-Fuentes, 1995). Para solventar estas deficiencias, se emplean los datos del padrón de 1924, y en algunos municipios se combinan estos con datos de empresa. El padrón está disponible, en general, para todos los municipios españoles, y en él se recogen los datos por hogares y también por cada individuo con una detallada información sociodemográfica que contiene las ocupaciones, lo que nos permite calcular las tasas de actividad tomando en consideración distintas variables: edad, estado civil, número de hijos, salarios de los miembros del hogar, etc. ${ }^{2}$.

En la Tabla 1 presentamos la TAF a nivel provincial y municipal, contrastando los datos del censo de 1920 y los del padrón de 1924 para los dos municipios urbanos seleccionados; no ha sido posible realizar esta comparación para los ayuntamientos rurales,

1. En concreto, en el grupo 70 se contabilizan los individuos momentáneamente sin ocupación; en el 71, alumnos de escuelas y colegios de primera enseñanza; en el 72, estudiantes; en el 73, acogidos en hospicios y en hospitales; en el 74, locos y enajenados; en el 75, presos y presidiarios; en el 76 , mendigos, vagabundos y prostitutas; en el 77, individuos sin profesión; en el 78, miembros de la familia; y en el 79, niños sin profesión por razón de su edad. El 80 se refiere a profesión desconocida o sin especificar.

2. En concreto, la información recogida para cada miembro del hogar es la siguiente: 1) nombre y apellidos; 2) sexo; 3) fecha y lugar de nacimiento (municipio, provincia); 4) nación; 5) estado civil; 6) parentesco o razón de convivencia con el cabeza de hogar; 7) ¿sabe leer, escribir?; 8) ocupación principal o modo de vivir; 9) residencia legal; 10) tiempo que lleva viviendo en el ayuntamiento donde se inscribe; 11) sitio donde se hallan los ausentes; 12) clasificación vecinal de los habitantes; 13) renta anual o jornal. 
pues, como hemos señalado, el censo sólo ofrece información detallada para las provincias y sus capitales. A partir de la información censal calculamos la TAF dividiendo el número de mujeres ocupadas entre el total de mujeres comprendidas entre los 11 y los 70 años. A partir de los datos del padrón de 1924 calculamos la TAF dividiendo el número de mujeres ocupadas entre 15 y 64 años y el total de mujeres de este tramo de edad ${ }^{3}$. Nuestros datos provienen de una muestra aleatoria compuesta por 15.602 habitantes y 3.316 hogares vaciados del padrón de 1924. La muestra es menor para las ciudades que para los pueblos (10\% y 30\%-50\%, respectivamente), pero significativa según estudios realizados por especialistas en demografía histórica e historia urbana para el País Vasco (González Portilla, 2009). La muestra se distribuye de la siguiente forma: A Coruña, 1.531 hogares y 6.672 individuos; Ourense, 360 hogares y 2.171 individuos; Bueu, 471 hogares y 2.172 individuos; Padrón, 810 hogares y 3.845 individuos; y, por último, Nigrán, 750 individuos y 144 hogares ${ }^{4}$. Con base a estos datos se ha corregido el subregistro femenino del padrón de 1924 en la agricultura, las industrias marítimas y el pequeño comercio. En la agricultura, en concreto, siguiendo una metodología similar a la efectuada en el País Vasco, imputamos el oficio de labradora a las esposas e hijas de labradores que figuraban sin oficio (Pérez-Fuentes, Pareja \& Zarraga, 2010). En la pesca se imputa la ocupación de auxiliar de pesca a las esposas de pescadores, ya que usualmente se dedicaban a la limpieza de barcos, elaboración de comida para la tripulación, fabricación y reparación de redes, marisqueo, recolección de algas, etc. En la industria conservera se han cruzado los datos de población con los de algunas empresas: expedientes de trabajadores y libros de salarios de la fábrica Massó Hermanos y matrícula de operarios de Antonio Alonso. Así se descubre que muchas de las que figuraban en el padrón como sus labores trabajaban en las fábricas de conservas y salazones (Muñoz Abeledo, 2010) ${ }^{5}$. También, combinando los datos del padrón con los de los registros municipales de vendedores de la lonja de pescado y del mercado de abastos, se saca a la luz más mujeres ocupadas fuera del hogar. Por último, en el sector terciario, a las esposas de los pequeños comerciantes (panaderías, bo-

3. En el censo de 1920 la población ocupada aparece dividida en tres grupos de edad: menores de 21 , entre 21 y 60 , y mayores de 60 . El cálculo de la tasa de actividad se ha realizado incluyendo en el numerador a las menores de 21 años, porque era habitual que comenzasen a trabajar a partir de los 10-11 años en el mundo urbano (MuÑoz Abeledo, TABOAdA \& Verdugo, 2015). También se incluye a las mayores de 60 , aunque en realidad en estas edades había muy pocas ocupadas. Este número se divide entre las mujeres de edades comprendidas entre 11 y 70 años, ya que es el que más se asemeja al considerado como población potencialmente activa en la actualidad (15-64 años). Además, esto permite comparar los resultados obtenidos con los de otras publicaciones recientes indicadas en la introducción y que utilizan estos grupos etarios.

4. En el caso de Nigrán el vaciado ha sido menor (10\%), similar al de las ciudades debido a que por su carácter agrario no había apenas variación en las ocupaciones.

5. Borderías (2002), Borderías y LóPEz GuAllar (2003) y MuÑoz AbEledo (2010) adjudican actividad a las obreras de las fábricas cuya ocupación no consta en el padrón. 
degones, fondas, etc.) se les asigna el mismo oficio que tenían sus maridos, pues habitualmente se trataba de negocios familiares ${ }^{6}$. Aplicando esta metodología se consigue atribuir un oficio a 712 mujeres de las 3.582 que en la muestra figuraban sin ocupación.

TABLA 1

Tasa de actividad femenina

\begin{tabular}{|c|c|c|c|c|c|}
\hline \multicolumn{2}{|c|}{ Provincias } & \multicolumn{4}{|c|}{ Municipios } \\
\hline & Censo 1920 & & Censo 1920 & Padrón 1924 & Padrón 1924(corregido) \\
\hline A Coruña & $26 \%$ & A Coruña & $22 \%$ & $26 \%$ & $33 \%$ \\
\hline Ourense & $14 \%$ & Ourense & $15 \%$ & $38 \%$ & $46 \%$ \\
\hline Lugo & $22 \%$ & Bueu & - & $37 \%$ & $69 \%$ \\
\hline \multirow[t]{2}{*}{ Pontevedra } & $35 \%$ & Padrón & - & $58 \%$ & $64 \%$ \\
\hline & & Nigrán & - & $4 \%$ & $65 \%$ \\
\hline
\end{tabular}

Fuente: elaboración propia a partir del censo de 1920 y del padrón de 1924.

Como se observa en la Tabla 1, la tasa de actividad femenina por provincias se asemeja mucho a la de los municipios urbanos considerados. Destaca también que las provincias litorales, A Coruña y Pontevedra, presentan una mayor ocupación femenina que las del interior, lo cual concuerda con el modelo de industrialización, muy centrado en la pesca y su transformación, que tuvo lugar justo en las primeras décadas del siglo Xx (Carmona, 1997; Carmona \& Nadal, 2005). Además, si se comparan a nivel municipal los datos de actividad que se obtienen mediante una y otra fuente (censo y padrón), se observa que, tras realizar la corrección, Ourense más que duplica su tasa de actividad mientras que la tasa de A Coruña únicamente se ve incrementada en cuatro puntos porcentuales. Como se explicará con más detalle en el siguiente epígrafe, ello es debido a que en Ourense predominaba el sector agrario, por lo que después de la corrección se visibiliza a las labradoras de esta ciudad. Finalmente, si se comparan los datos sin corregir y corregidos para los municipios rurales, se ve claramente el incremento de la TAF en todos ellos debido a la presencia de esposas e hijas de labradores (Nigrán y Padrón) y de pescadores (Bueu), que figuraban sin ocupación y a las que se les ha asignado el oficio correspondiente, tal y como se ha indicado.

En definitiva, el artículo ofrece resultados de las tasas de actividad femenina por edad, estado civil y número de hijos. Pero además, propone un modelo estadístico para explorar los determinantes de oferta y demanda que podrían condicionar la participación la-

6. PÉrez-Fuentes $(1993,2003)$ adjudica actividad de servicios a las esposas e hijas mayores de 15 años sin ocupación en el padrón en los hogares donde el cabeza de familia es jornalero, y residen huéspedes jornaleros. 
boral de las mujeres casadas, decantándose por formular un modelo GAM (modelo aditivo generalizado $)^{7}$, aplicado a la muestra de población con respuesta binaria: trabaja/no trabaja. La variable dependiente es aquí la participación de las casadas en el mercado laboral y las variables explicativas consideradas son: municipio de residencia, salario del marido, ocupación del marido, maternidad, número de hijos, hijos menores de 5 años, hijos trabajando, número de ocupados del hogar. Hemos elegido un modelo GAM en lugar de un modelo lineal generalizados (GLM), porque es más flexible y más eficaz para trabajar con relaciones no lineales. Los modelos GAM reemplazan el predictor lineal por una suma de funciones suaves, de tal forma que se pueden emplear en el modelo variables explicativas continuas (salario) y categóricas (ocupación).

\section{LOS MERCADOS DE TRABAJO EN EL MUNDO URBANO Y RURAL}

En la Galicia de principios del siglo xx, las diferentes estructuras económicas del campo y la ciudad condicionaban que los mercados de trabajo rural y urbano fueran muy distintos, tanto para hombres como para mujeres, tal y como se describe a continuación. El mercado laboral de las ciudades consideradas en este estudio presenta algunas diferencias, sobre todo en la agricultura y en la industria. En A Coruña, la importancia del puerto, el auge de la navegación a vapor y la existencia del ferrocarril A Coruña-Madrid impulsaron el desarrollo económico, especialmente en el comercio (Blanco, 2001; Mirás, 2004; Lindoso \& Mirás, 2001). Ourense es la típica capital de provincia en la que la economía se basa en el comercio y los servicios, aunque la agricultura tenía un peso nada desdeñable, tanto en la ciudad como en sus alrededores ${ }^{8}$. Según el censo de la población de España de 1920 el sector más desarrollado en ambas ciudades era el terciario, con casi la mitad de la ocupación, seguido del secundario y del primario ${ }^{9}$. En A Coruña el sector primario estaba vin-

7. El modelo aditivo generalizado, presentado por HASTIE y TiBSHIRANI (1990), es una extensión de los modelos tradicionales de regresión lineal para el análisis de los datos que incorpora la no linealidad y la regresión no paramétrica. El modelo está construido por la suma de funciones suaves de las variables predictoras, pudiendo ser estas variables continuas, categóricas, número de casos y series de datos. Entre las bondades de los modelos GAM está que no es necesario que las variables tengan una distribución normal; el usuario no tiene que sugerir el tipo de función existente entre las variables, sino que es el modelo quien define la forma de esta relación.

8. En ambas capitales la burguesía dinamizaba los servicios, el comercio y la industria. Resulta destacable que la mayor parte de ellos sean foráneos, nacidos en Castilla, Asturias, Cataluña y Euskadi. Por ejemplo, en Ourense muchos arrieros de Logroño, Zamora, Valladolid o Asturias, que antaño pasaban por Ourense para ejercer su profesión, se quedaban definitivamente en la capital como almacenistas y comerciantes (Lindoso \& MirÁs, 2001; Somoza, 2000, 2001). Se trata de lo que OTERo (1973) denominó colonización comercial (SOMOZA, 2000, 2001; VALCÁRCEL, 1999).

9. El porcentaje de ocupados en el sector terciario era del $46,2 \%$ en A Coruña y del $49,4 \%$ en Ourense; el secundario representaba el 45,5\% de la ocupación de A Coruña y el 31,8\% en Ourense; 
culado al mar, concentrándose su puerto en el desembarco y la comercialización de la pesca en fresco (Carmona, 1997; Giráldez, 1996), mientras que el de Ourense se vinculaba a la agricultura, cuyos productos se destinaban a satisfacer la demanda urbana (Somoza, 2001).

En cuanto al sector secundario, basándose en la contribución industrial y de comercio de 1924, el porcentaje de empresarios coruñeses dedicados a actividades artesanales y a la industria respecto del total de contribuyentes era del $18 \%$ y del $26 \%$, respectivamente. La industria más importante era la de tabaco, donde el trabajo era realizado básicamente por mujeres. La industria alimentaria de la ciudad de A Coruña estaba constituida por varias fábricas de salazón y de conservas que incluso exportaban a los mercados de Cuba y Puerto Rico. El sector de transformación de pescado, de gran tradición en la ciudad, tuvo un importante desarrollo y ejerció un efecto de arrastre sobre otras industrias, como las metalográficas, las de envases y las de hielo ${ }^{10}$. Otra industria singular donde trabajaban mujeres era la de las fábricas de sombreros, cuyos productos alcanzaron gran fama en el extranjero, incluso una de ellas fue premiada varias veces en las exposiciones universales de Londres y París ${ }^{11}$. Respecto de la industria orensana, de acuerdo a la contribución industrial, en 1916 había un total de 31 licencias industriales y 570 matrículas. Destacaban los siguientes subsectores: el de la alimentación, el metalmecánico y el auxiliar de la construcción, creados en su mayoría por foráneos. Como era normal en las pequeñas capitales de provincia, la industria alimentaria orensana la constituían los molinos harineros, las fábricas de chocolate y las fábricas de hielo y gaseosas. Por su parte, el subsector metal-mecánico de Ourense se originó a partir de la producción de hierro de la fundición Malingre e influyó de manera decisiva en la creación de otros talleres que desarrollaron la misma actividad.

Finalmente, con respecto al sector terciario, A Coruña era una ciudad eminentemente comercial en el primer tercio del siglo xx (Precedo, 1990). De hecho, según datos de la contribución industrial y de comercio de 1924, el 32\% de los empresarios eran comerciantes y el $24 \%$ se enmarcaban dentro de actividades incluidas en otros servicios. El pequeño comercio (tiendas de alimentación, de textiles, de quincalla o mercerías) empleaba a un número importante de mujeres. El dinamismo comercial incrementaba la oferta de servicios, como los de hostelería, cafeterías, restaurantes, aprovisionamiento de víveres,

el primario era del 8,3\% en A Coruña y del 18,8\% en Ourense.

10. También existían numerosas fábricas de harina, panificadoras y fábricas de chocolate, fábricas de cerveza o destilerías, y una fábrica de fideos y pasta para sopa (SomozA, 2001).

11. También había numerosos telares para lienzo manuales, una fábrica de pasamanería y cintas, y dos de peines, al menos tres de cerillas, varias fábricas de jabón-cuya producción era exportada en gran parte a América-, una de algodón para mantas, otra de hules, una de azogar espejos, una de marcos dorados, otra de puntas de París, y múltiples zapateros o carpinteros, etc. (SomOzA, 2001). 
etc. (Mirás, 2004; Lindoso \& Mirás, 2001). En Ourense, el comercio se sustentaba en tres pilares: los tejidos, los almacenes de alimentación y las ferreterías, que suministraban sus productos a toda la provincia ${ }^{12}$.

Una vez explicada la estructura económica de estas dos urbes, se va a analizar su mercado laboral a partir de los datos del padrón de 1924. Según esta fuente, se evidencia el mayor nivel de industrialización de A Coruña frente a Ourense: en la primera predominaban los jornaleros industriales, mientras que en la segunda lo hacían los ocupados en oficios tradicionales (Tabla 2). Por lo que respecta a las mujeres, en ambas ciudades se empleaban mayoritariamente en el sector servicios, fundamentalmente como criadas; la segunda ocupación de las coruñesas eran las fábricas y la de las orensanas los oficios tradicionales; por último, en ambas urbes en torno al 10\% de las mujeres se ocupaba en la hostelería y el comercio.

TABLA 2

Distribución porcentual de la ocupación en A Coruña y Ourense

\begin{tabular}{lcccc}
\hline & \multicolumn{2}{c}{ A Coruña } & \multicolumn{2}{c}{ Ourense } \\
& Hombres & Mujeres & Hombres & Mujeres \\
\hline Labradores y jornaleros & $2 \%$ & $1 \%$ & $10 \%$ & $9 \%$ \\
Marineros y pescadores & $4 \%$ & $6 \%$ & $0 \%$ & $0 \%$ \\
Jornaleros industriales & $45 \%$ & $26 \%$ & $10 \%$ & $1 \%$ \\
Oficios tradicionales & $11 \%$ & $15 \%$ & $25 \%$ & $27 \%$ \\
Trabajadores de los servicios & $3 \%$ & $32 \%$ & $4 \%$ & $39 \%$ \\
Hostelería y comercio & $11 \%$ & $12 \%$ & $18 \%$ & $13 \%$ \\
Profesiones liberales & $9 \%$ & $3 \%$ & $21 \%$ & $7 \%$ \\
Militares & $11 \%$ & $0 \%$ & $6 \%$ & $0 \%$ \\
Iglesia & $0 \%$ & $3 \%$ & $0 \%$ & $3 \%$ \\
Rentistas y propietarios & $4 \%$ & $1 \%$ & $6 \%$ & $1 \%$ \\
\hline Total & $\mathbf{1 0 0} \%$ & $\mathbf{1 0 0} \%$ & $\mathbf{1 0 0} \%$ & $\mathbf{1 0 0} \%$ \\
\hline
\end{tabular}

Fuente: elaboración propia a partir de los datos del padrón de 1924.

12. Los de alimentación, que incluían comestibles, carnes frescas, pescado, aceite y vinagre, alcanzaban el número de 75 establecimientos; los de tejidos (camisería, ropa hecha, calzado, sombreros, encajes y alfombras), 54; las ferreterías (quincalla, cubiertos y cacharros) eran 19; las tiendas de joyería y relojería, 8; las librerías, 7 ; las mercerías, 5; las tiendas de harina, 4 ; las de curtidos, 4 ; los establecimientos de vino, 3; las droguerías, 2; y otros (objetos eléctricos, máquinas de coser, monturas, armas, azulejos, juguetes, carbón y paja), 15. Todo ello, sin contar con las matrículas de las pensiones, hoteles, tabernas, cafés y figones (SomozA, 2001). 
En las zonas rurales del norte de España, al igual que acontecía en otras partes de Europa, las explotaciones agrarias se caracterizaban por su pequeña dimensión y por la utilización de trabajo familiar, lo que implicó unos altos índices de participación femenina (Grantham \& Grimard, 2010). En la Galicia de comienzos del siglo xx era habitual que las esposas y las hijas de los labradores trabajaran como labradoras, ocupación que mantenían a lo largo de su vida. En Nigrán y en Padrón la agricultura era la principal ocupación, tanto para hombres como para mujeres (Tabla 3). Los oficios tradicionales eran la segunda ocupación masculina en Padrón (20\%), donde se incluían albañiles, canteros, carpinteros, etc., pero con un peso elevado de sastres ${ }^{13}$, mientras que para las mujeres la segunda actividad era la de sirvienta. Además, este municipio estuvo fuertemente afectado desde la segunda mitad del siglo xIx por la emigración, mayoritariamente masculina, lo que favoreció el acceso de las mujeres al trabajo (Muñoz Abeledo, Taboada \&Verdugo, 2015), fundamentalmente en las labores del campo.

TABLA 3

Distribución porcentual de la ocupación en Bueu, Padrón y Nigrán

\begin{tabular}{lrrrrrr}
\hline & \multicolumn{2}{c}{ Bueu } & \multicolumn{2}{c}{ Padrón } & \multicolumn{2}{c}{ Nigrán } \\
& Hombres & Mujeres & Hombres & Mujeres & Hombres & Mujeres \\
\hline Labradores y jornaleros & $2 \%$ & $2 \%$ & $45 \%$ & $70 \%$ & $60 \%$ & $88 \%$ \\
Marineros y pescadores & $54 \%$ & $27 \%$ & $2 \%$ & $1 \%$ & $8 \%$ & $6 \%$ \\
Jornaleros industriales & $22 \%$ & $62 \%$ & $4 \%$ & $6 \%$ & $0 \%$ & $0 \%$ \\
Oficios tradicionales & $6 \%$ & $0 \%$ & $20 \%$ & $2 \%$ & $7 \%$ & $0 \%$ \\
Trabajadores de los servicios & $1 \%$ & $3 \%$ & $3 \%$ & $15 \%$ & $3 \%$ & $3 \%$ \\
Hostelería y comercio & $8 \%$ & $5 \%$ & $8 \%$ & $4 \%$ & $3 \%$ & $2 \%$ \\
Profesiones liberales & $3 \%$ & $1 \%$ & $14 \%$ & $1 \%$ & $20 \%$ & $0 \%$ \\
Militares & $1 \%$ & $0 \%$ & $2 \%$ & $0 \%$ & $0 \%$ & $0 \%$ \\
Rentistas y propietarios & $3 \%$ & $0 \%$ & $2 \%$ & $1 \%$ & $1 \%$ & $0 \%$ \\
\hline Total & $\mathbf{1 0 0} \%$ & $\mathbf{1 0 0} \%$ & $\mathbf{1 0 0} \%$ & $\mathbf{1 0 0} \%$ & $\mathbf{1 0 0} \%$ & $\mathbf{1 0 0} \%$ \\
\hline
\end{tabular}

Fuente: elaboración propia a partir de los datos del padrón de 1924.

A diferencia de los municipios anteriores, la actividad económica de Bueu giraba en torno a la pesca y su transformación, donde se empleaba a más de las tres cuartas partes de la población activa. Los hombres eran predominantemente pescadores, aunque también trabajaban en tareas de elaboración de envases y reparación de maquinaria en la industria conservera. La transformación del pescado ofrecía a las mujeres empleo temporal, tal y como acontecía en todo el litoral norte y noroeste de España a lo largo de los siglos XIX

13. En la muestra, el $30 \%$ de los hombres incluidos en oficios tradicionales son sastres. 
y Xx, ya fuese la sardina, como en el caso gallego, o la anchoa, en el Cantábrico ${ }^{14}$. Otras actividades auxiliares de la pesca, como el marisqueo, el preparado del pescado, su transporte, su venta, la reparación de las redes, la limpieza de los barcos o la elaboración de comida para la tripulación, también eran actividades habituales para las esposas y las hijas de los pescadores ${ }^{15}$.

En suma, las principales ocupaciones de hombres y mujeres en el rural eran agricultor/a y jornalero/a, con la excepción del litoral, donde las industrias marítimas empleaban a los hombres en la pesca y a las mujeres en las fábricas de procesado de pescado. Por lo que respecta al mundo urbano, la principal ocupación femenina era el servicio doméstico, mientras que los hombres se ocupaban en las industrias y oficios tradicionales.

\section{TASAS DE ACTIVIDAD FEMENINA: FACTORES DE OFERTA}

Una vez que se han analizado los condicionantes de demanda del trabajo femenino, en este apartado se examina la influencia que tienen los factores de oferta sobre él. Para ello se calcula en cada uno de los cinco municipios de nuestro estudio la TAF por edad, estado civil y, para las casadas, la TAF según el número de hijos, hijos menores de 5 años, hijos ocupados y salario del esposo.

Con relación a la TAF por edad, se observa que toma valores más elevados en los municipios rurales que en los urbanos (Gráfico 1). Dentro de los rurales es Bueu el que presenta las tasas más altas, alcanzando el $70 \%$ desde los 25 años y manteniéndose así a lo largo del ciclo vital, algo en consonancia con la estructura productiva del municipio, muy centrada en las industrias marítimas. A continuación, se encuentra Padrón, donde se combina el trabajo agrario complementado con el manufacturero en la industria textil.

14. En el municipio de Bueu había 17 fábricas de salazón y en la parroquia más costera, San Martín de Bueu, un total de 9 (SÁnchez Cidrás, CERviÑo \& FERnández Aldegunde, 1998). Encontramos más ejemplos a lo largo de la geografía gallega: en 1847 la ría de Ares contaba con poco más de 1.900 habitantes y unas 700 mujeres que trabajaban en las fábricas de salazón («Pesquerías de Galicia", Boletín Mercantil e Industrial de Galicia, n. ${ }^{\circ}$ 9, 20/11/1847); a finales del siglo xIx, en la ría de Muros había 28 fábricas de salazones y conservas en las que trabajaban varios miles de hombres y de mujeres (ARTAZA, 1959: 175). En otras localidades españolas las fábricas de salazón proporcionaron empleo a un gran número de mujeres, como en el caso del País Vasco (Homobono, 1993) y otros municipios del Cantábrico, en donde la salazón empleaba casi exclusivamente mano de obra femenina e infantil (ANSOLA, 1996).

15. Lo mismo sucedía en otras localidades pesqueras del Atlántico y del Pacífico, tal y como lo constata Pettersen (1996) para las islas noruegas de Lofoten y CoOPER (1992) para British Columbia. Para el caso de Bueu, se puede consultar MuÑoz Abeledo (2002, 2010). 


\section{GRÁFICO 1}

Tasa de actividad femenina por edad

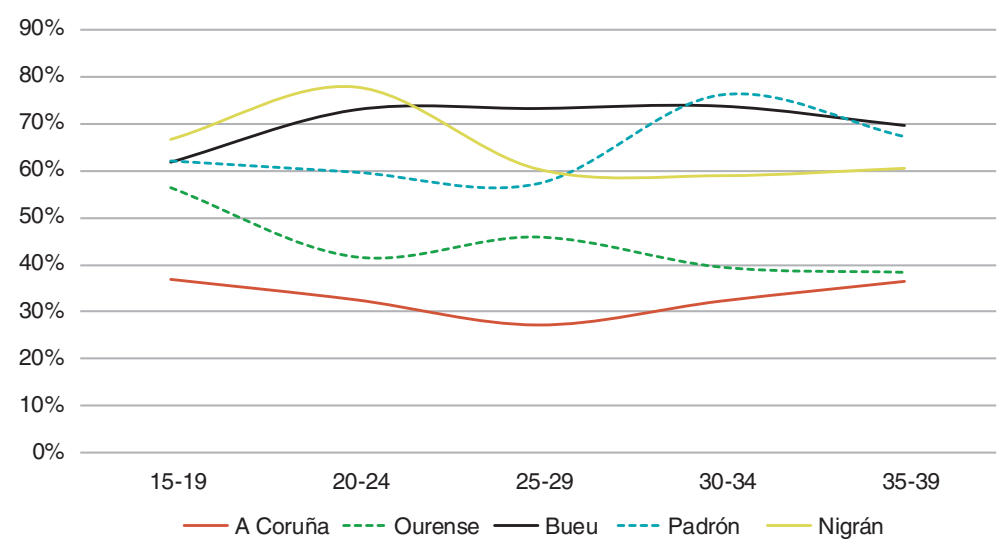

Fuente: elaboración propia a partir de los datos del padrón de 1924.

Los municipios urbanos de A Coruña y Ourense tienen una TAF más reducida que los rurales. En A Coruña, esta es bastante estable (entre el 30\% y el 40\%), hecho que se justifica por el importante peso de las mujeres en la industria, al ser una cuarta parte de las trabajadoras jornaleras industriales, muchas de ellas empleadas en la Fábrica de Tabacos, lo que les daba una mayor estabilidad laboral a lo largo de su vida. En Ourense la tasa alcanza casi el $60 \%$ para las menores de 25 años, y se sitúa entre el $40 \%$ y el $50 \%$ en el resto de las edades ${ }^{16}$. Estos datos resultan paradójicos teniendo en cuenta que A Coruña era una ciudad más dinámica que Ourense, pero se explican por el peso relativamente elevado del sector agrario, en el cual la ocupación femenina ha sido corregida sobre los datos del padrón, como ya hemos explicado en la metodología. Si comparamos nuestros cálculos de las tasas de actividad por edad con los datos obtenidos para 1920 en Cataluña, observamos que en las edades más jóvenes (14-24 años) la TAF catalana es superior a la gallega, pero en los siguientes grupos de edad es más elevada en los municipios rurales gallegos. Esto es debido a las diferentes estrategias familiares, pues en Cataluña las mujeres se retiraban del mercado laboral cuando sus hijos trabajaban, tal y como veremos a continuación $^{17}$.

16. En A Coruña, está cerca del $40 \%$ en el tramo de 15-24 años, y sufre una leve pero continuada reducción hasta los 44 años, edad en la que la tasa de actividad está por debajo del 30\%; a partir de los 45 años experimenta una suave pero constante subida hasta alcanzar valores próximos al $40 \%$. En Ourense, el tramo de 15-24 años presenta una tasa de casi el $60 \%$, que se reduce hasta el $40 \%$ en las edades superiores a los 45 .

17. En el textil catalán las tasas de actividad son altas (por encima del $70 \%$ ) entre los 15 y los 39 años, y se reducen a partir de los 40 años hasta situarse por debajo del $24 \%$ a partir de los 50 años (BORDERÍAS \& FERRER, 2015). 
Lo primero que se observa al calcular la TAF por estado civil es que las solteras trabajaban más en las ciudades mientras que las casadas y las viudas lo hacían en el rural (Gráfico 2). La baja TAF de las casadas urbanas se explica por dos motivos; en primer lugar, porque la ocupación mayoritaria de las mujeres era la de criadas, actividad que abandonaban cuando se casaban; en segundo lugar, porque en las ciudades no hemos podido corregir completamente el subregistro de los datos del padrón. En contraposición, las mujeres del rural eran fundamentalmente agricultoras, independientemente de su estado civil, ya que la estructura de la propiedad agraria en Galicia propiciaba el trabajo de todos los miembros de la familia (Villares, 1982; Freire, 2008). Además, en el litoral gallego la TAF era alta por la existencia de industrias del mar.

\section{GRÁFICO 2}

\section{Tasa de actividad femenina por estado civil}

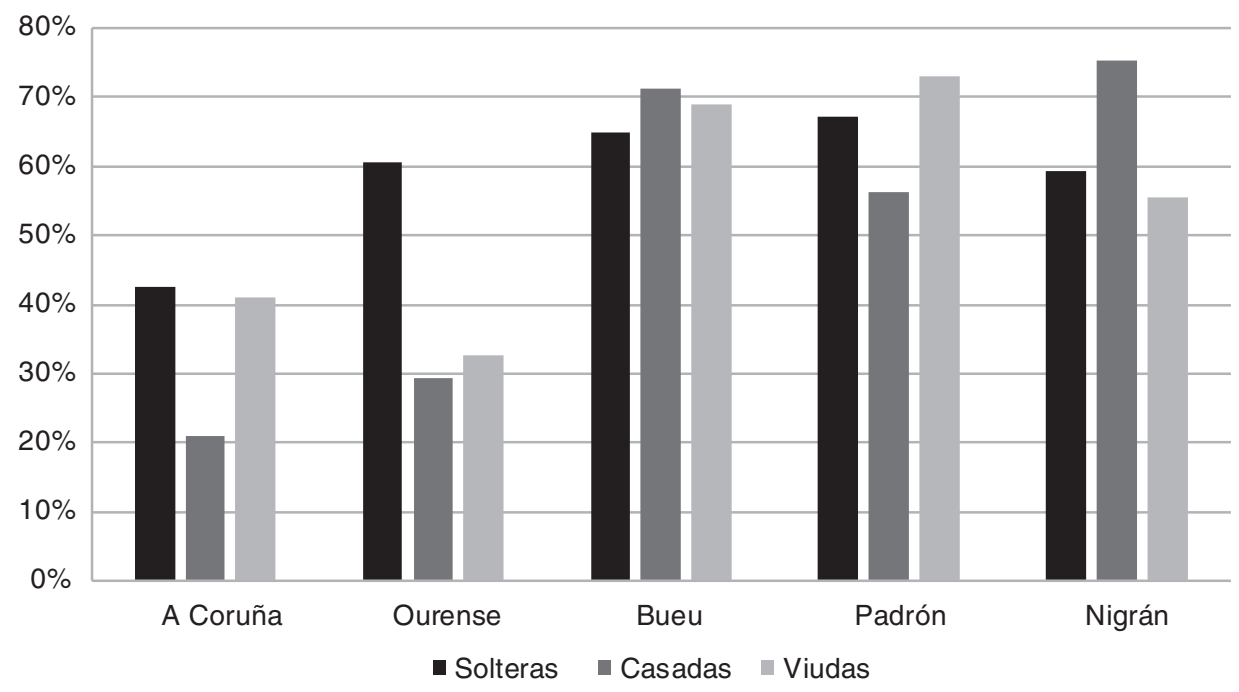

Fuente: elaboración propia a partir de los datos del padrón de 1924.

Cuando se comparan estos resultados con los obtenidos en Cataluña ${ }^{18}$ se observa que las solteras gallegas presentan tasas de actividad inferiores a las catalanas, mientras que son superiores en las casadas y viudas. Estos resultados se explican porque en Cataluña las familias del textil liberaban a las casadas del trabajo en la fábrica a medida que se incorporaban los activos más jóvenes del hogar; sin embargo, las familias de la conserva gallega mantenían a las casadas trabajando temporalmente, incluso cuando los hijos se incorporaban al mercado laboral.

18. En 1920 en los municipios textiles catalanes la TAF de las solteras era del 86,3\%, la de las casadas, del 55,4\% y la de las viudas, del 32,8\% (BordERÍAs \& FERRER, 2015, 2017). 
Para las casadas, se ha calculado también su actividad según el número de hijos con el fin de analizar si la maternidad truncaba su vida laboral (Gráfico 3). En el caso de las ciudades no variaba en función del número de hijos la tasa de actividad, que se mantenía entre un $20 \%$ y un $30 \%$. En las pequeñas villas con industria esta era elevada con independencia del número de hijos: 50\% en Padrón y 70\% en Bueu. Por último, en Nigrán superaba el $80 \%$ debido al elevado peso del sector agrícola, dominado por el minifundio familiar.

\section{GRÁFICO 3}

Tasa de actividad de las casadas según número de hijos

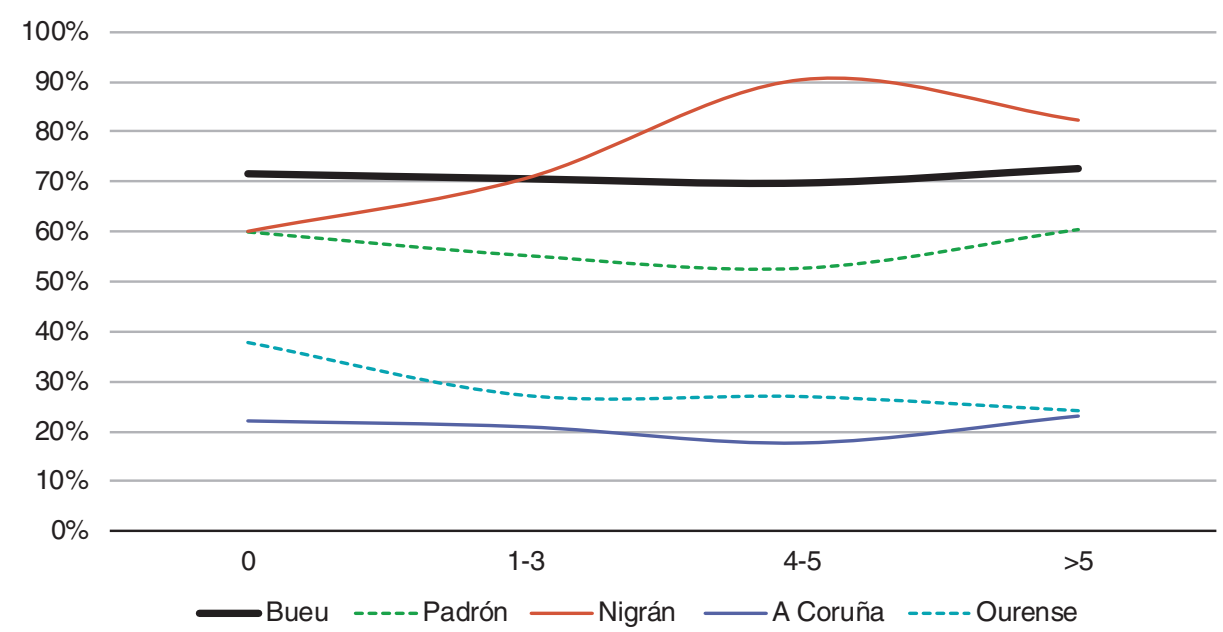

Fuente: elaboración propia a partir de los datos del padrón de 1924.

Por lo que se refiere a la tasa de actividad de las casadas con hijos que trabajan, en los municipios rurales oscila entre el $80 \%$ de Bueu y casi el $70 \%$ de Padrón (Gráfico 4). Por lo tanto, la maternidad no significaba un abandono del mercado laboral; es más, incluso se observa que la TAF aumenta cuando hay tres o más hijos en el hogar. Posiblemente, las madres dejarían a los más pequeños al cuidado de otros miembros de la familia (hijos mayores, abuelas, etc.) o de una muchacha (Muñoz Abeledo, 2010), estrategia diferente a la observada en Cataluña ${ }^{19}$. En el caso de las ciudades, la TAF de las casadas se mantiene en torno al $30 \%$, independientemente del número de hijos, valor próximo al de la tasa global femenina de estas urbes (Tabla 1).

19. En la década de 1920, en la Cataluña textil la edad de acceso al matrimonio se situaba en torno a los 26 años; a partir del grupo de edad de 36-40 se observa una disminución de la tasa de actividad de las casadas, que coincide con la incorporación de sus hijos al trabajo en las fábricas en sustitución de sus madres (BORDERÍAs \& FERRER, 2015). 


\section{GRÁFICO 4}

Tasa de actividad de las casadas con hijos que trabajan

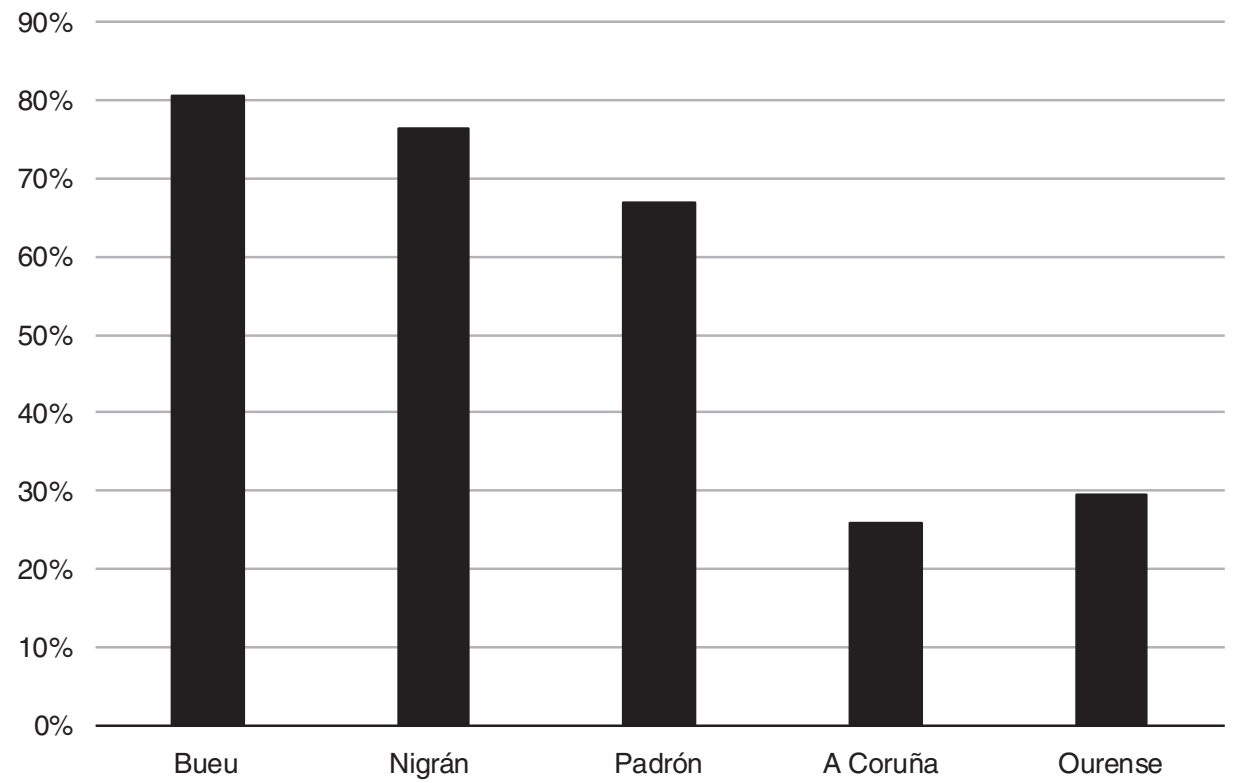

Fuente: elaboración propia a partir de los datos del padrón de 1924.

Para completar el análisis de los condicionantes de la actividad de las casadas, a continuación, se propone un modelo estadístico (modelo aditivo generalizado, GAM) (Wood, 2017), aplicado a la muestra de población con respuesta binaria: trabaja/no trabaja. En dicho modelo se introdujeron diferentes variables explicativas de oferta (salario y ocupación del esposo, edad de la mujer y del marido, número de hijos, hijos menores, hijos ocupados y número de personas ocupadas en el hogar) y de demanda laboral (municipio de residencia) que podrían condicionar la actividad femenina (Tabla 4). A continuación se presentan los resultados del modelo finalista seleccionado ${ }^{20}$.

Por el lado de la demanda laboral, la variable municipio de residencia condiciona la probabilidad de trabajar de las casadas, pues es Bueu, con sus empresas de transformados marítimos, el municipio que representa la industrialización del litoral de la región y el que, justamente, obtiene un coeficiente positivo (Tabla 4). Por el lado de la oferta, los factores que influían en la actividad de las casadas eran los siguientes: edad de la mujer, edad del esposo, número de hijos, hijos menores de 5 años, hijos que trabajan, número

20. Además de las variables incluidas en el modelo finalista, se excluyeron, por no ser significativas, las siguientes: ser madre (valor 1 si la mujer es madre y 0 en otro caso) y número de personas que componen el hogar. 
TABLA 4

Modelo GAM-logit de la actividad de las casadas en Galicia

\begin{tabular}{|c|c|c|c|c|c|}
\hline Coeficientes paramétricos lineales & Estimador & Error estandar & Z valor & $\operatorname{Pr}(>|z|)$ & \\
\hline (Intercept: Bueu) & 2,369 & 0,557 & 4,253 & $2,11 e-05$ & $* * *$ \\
\hline Salario diario del esposo & $-0,004$ & 0.002 & $-2,099$ & 0,036 & * \\
\hline \multicolumn{6}{|l|}{ Ocupación del esposo } \\
\hline Labrador o jornalero agrícola & 1,485 & 0,519 & 2,861 & 0,004 & ** \\
\hline Marinero o pescador & 1,708 & 0,729 & 2,343 & 0,019 & * \\
\hline Jornalero industrial & $-1,384$ & 0,441 & $-3,136$ & 0,002 & ** \\
\hline Oficio tradicional & $-1,461$ & 0,473 & $-3,090$ & 0,002 & ** \\
\hline Servicios & $-3,446$ & 1,000 & $-3,445$ & 0,001 & $* * *$ \\
\hline Hostelería y comercio & 0,329 & 0,483 & 0,682 & 0,496 & \\
\hline Profesión liberal o empleado & $-2,898$ & 0,521 & $-5,559$ & $2,72 \mathrm{e}-08$ & $\star \star *$ \\
\hline Militar o cuerpos de seguridad & $-3,688$ & 0,944 & $-3,908$ & $9,31 e-05$ & $* * *$ \\
\hline Rentista, propietario o fabricante & $-2,907$ & 0,579 & $-5,017$ & $5,24 \mathrm{e}-07$ & $* * *$ \\
\hline Sin oficio & $-0,646$ & 0,789 & $-0,819$ & 0,413 & \\
\hline Ausente & 4,229 & 1,582 & 2,674 & 0,007 & ** \\
\hline Hijos $<5$ años & 0,274 & 0,247 & 1,107 & 0,268 & \\
\hline \multicolumn{6}{|l|}{ Municipio } \\
\hline A Coruña & $-2,528$ & 0,392 & $-6,446$ & $1,15 \mathrm{e}-10$ & $* * *$ \\
\hline Nigrán & $-2,115$ & 0,642 & $-3,297$ & 0,001 & $* * *$ \\
\hline Ourense & $-2,824$ & 0,454 & $-6,221$ & $4,93 e-10$ & $\star * *$ \\
\hline Padrón & $-2,361$ & 0,445 & $-5,308$ & $1,11 \mathrm{e}-07$ & $* * *$ \\
\hline Coeficientes paramétricos no lineales & Edf & Ref. df & Chi. cd & P-valor & \\
\hline Edad de la mujer & 3,839 & 4,775 & 13,12 & 0,021390 & * \\
\hline Edad del marido & 4,167 & 5,197 & 25,56 & 0,000133 & *** \\
\hline Núm. de hijos & 1,001 & 1,001 & 23,06 & $1,58 \mathrm{e}-06$ & $\star * *$ \\
\hline Núm. de hijos trabajando & 3,824 & 4,714 & 70,65 & $1,05 e-13$ & $\star * \star$ \\
\hline Núm. de ocupados del hogar & 7,057 & 7,806 & 61,90 & $1,68 \mathrm{e}-10$ & $* * *$ \\
\hline Núm. de ocupados del hogar/miembros del hogar & 6,216 & 7,181 & 68,27 & $4,21 \mathrm{e}-12$ & $* * *$ \\
\hline
\end{tabular}

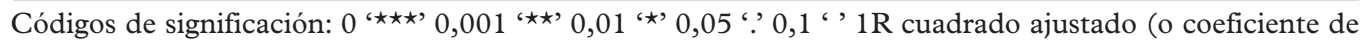
determinación ajustado) $=0,743$ Varianza explicada $=68,5 \%$ UBRE $=-0,54258 \quad$ Estimador de escala. $=1 \mathrm{n}=2048$

Nota metodológica: el tamaño muestral es $\mathrm{n}=2.048$. Las variables de tipo categórico del modelo son ocupación del marido y municipio, siendo el resto variables numéricas. El valor de la intercept o constante (2,369 en el modelo) recoge todas las categorías de referencia. Cada categoría del predictor se compara con la categoría de referencia.

UBRE es el parámetro de suavizado que minimiza un estimador del error cuadrático medio (MSE).

Fuente: Padrón de Población de 1924 para las localidades de A Coruña, Bueu, Nigrán, Ourense y Padrón. 
de ocupados en el hogar, ocupación y salario del esposo. En primer lugar, la actividad de las casadas aumenta con la edad, tal y como queda reflejado en el coeficiente positivo de esta variable. En segundo lugar, el número de hijos y los hijos ocupados no provocaban una retirada de las mujeres del mercado laboral, tal y como hemos constatado en la estadística descriptiva (Gráfico 4). Estos resultados, que ya conocíamos para las industrias marítimas (Muñoz Abeledo, 2010), se pueden extender ahora a los municipios de este estudio que representan otros modelos económicos (agricultura e industrias de textil y de tabaco). En tercer lugar, tanto el número de personas que trabajan como el coeficiente de personas ocupadas sobre el total de miembros del hogar aumentaban la probabilidad de que las casadas trabajasen. Esto se explica porque en Galicia, tanto en la agricultura como en la industria, las estrategias laborales de las familias pasaban por la ocupación del máximo número de activos para asegurar su supervivencia. En cuarto lugar, el modelo indica que la ocupación del cabeza de familia influía en la actividad de la esposa. En concreto, cuando este trabajaba en el sector primario (agricultores, jornaleros agrarios, pescadores, etc.) las mujeres también se ocupaban fuera del hogar. Además, el hecho de que el marido estuviese ausente -comúnmente en la emigración a ultramar-abocaba a las mujeres al trabajo; a la inversa, si el cabeza de familia era un trabajador cualificado (profesionales liberales, empleados, militares, etc.), esto incidía negativamente sobre la probabilidad de que las esposas trabajasen, tal y como indica el signo negativo de los coeficientes de las variables asociadas a estas profesiones. Por último, y en concordancia con lo anterior, el coeficiente de la variable salario diario del marido es negativo; es decir, que las mujeres de aquellos que tenían salarios más elevados permanecían inactivas ${ }^{21}$. Estos resultados fueron corroborados con un test de medias ${ }^{22}$ donde se comprobó si los salarios de los maridos de las casadas que trabajaban y de las que no trabajaban eran iguales o no, concluyéndose que si el esposo ganaba siete pesetas diarias o más, la esposa permanecía inactiva. Todos estos resultados confirman una de las cuestiones clave que se planteaban

21. En el caso de la utilización de los modelos de regresión logística existe la posibilidad de estimar las probabilidades teniendo en cuenta la interacción entre variables. El caso más claro sería: salario diario del esposo * ocupación del esposo; pero hemos probado a realizar la interacción y los resultados obtenidos no mejoran la variabilidad explicada del modelo. De ella extraemos que las profesiones de los esposos que resultan significativas con signo positivo son los oficios agrupados que representan a las clases trabajadoras: los trabajadores del sector primario, los oficios tradicionales y también algunos negocios familiares en los que las casadas intervendrían, como la hostelería y el comercio. En todas estas ocupaciones del esposo el signo es positivo con lo cual las esposas tienen más probabilidad de trabajar, como efectivamente se corresponde con las tasas de actividad y con los gráficos de ocupación presentados en los correspondientes apartados del artículo.

22. El test de comparación de medias se utiliza para decidir si las medias de dos poblaciones son iguales. En este caso, se dividió a las casadas en dos grupos (inactivas y activas) y se calculó el salario medio de los esposos de cada uno de los grupos, que dio valores muy diferentes $(7,04$ y 4,29 , respectivamente) como resultado, lo que indica que el salario del cabeza de casa influía en la actividad de la esposa. 
en la introducción de este artículo: que el salario de los esposos influía en la actividad de las mujeres casadas, de forma que la insuficiencia del salario del varón para sostener a la economía familiar se traduciría en altas tasas de actividad de las mujeres casadas, las cuales superan el $20 \%$ en A Coruña, el lugar donde se presentan más bajas por el subregistro y que ascienden a más del $70 \%$ en los municipios rurales y pesqueros. Por ello, se puede decir que el hombre no era el único ganador de pan en la Galicia de la década de 1920.

\section{CONCLUSIONES}

En este artículo se han planteado los condicionantes de la participación laboral de las mujeres y se ha calculado la tasa de actividad femenina en varias localidades de Galicia en el año 1924, lo que ha proporcionado valores que superan el $50 \%$ en el mundo rural, tanto en pueblos con industria (Bueu, 69\%, y Padrón, 64\%) como en municipios eminentemente agrarios (Nigrán, 65\%). Efectivamente, la estructura económica de la región, dominada por la agricultura, la pesca y su transformación, condicionaba el trabajo de las mujeres. En el caso de la industria, esencialmente localizada en el litoral, estos resultados son fruto de la combinación de fuentes demográficas (padrón de 1924) con documentación de empresa (Massó Hermanos, SA y Antonio Alonso). En la agricultura, donde el subregistro era muy alto, hemos corregido el padrón de 1924, asignando actividad a las mujeres e hijas de labradores que aparecían de ocupación sus labores en esta fuente. En las ciudades las tasas de actividad eran inferiores a las de los pueblos con industria: A Coruña el 33\% y Ourense el 46\%. Paradójicamente, la TAF era más elevada en Ourense, hecho atribuible al mayor peso de la agricultura en su cinturón urbano, así como a la ausencia de censos obreros, lo que no permite captar el subregistro de la industria coruñesa, que tenía mucho más peso que la orensana. La TAF así obtenida resuelve parcialmente el problema del subregistro y abre nuevas posibilidades a diferentes disciplinas que analicen el cambio estructural y la modernización económica regional.

En cuanto a los condicionantes de la actividad femenina, el principal factor impulsor de la demanda de empleo fue la consolidación del sector de conservas de pescado. Efectivamente, es el municipio de Bueu, como ejemplo paradigmático de este modelo industrial, el que presenta mayores tasas de actividad, algo que hemos contrastado estadísticamente, pues el modelo que presentamos en este artículo indica que residir en Bueu aumentaba la probabilidad de trabajar de las mujeres casadas. Además de la industria alimentaria, la agricultura ocupaba a miles de mujeres en la región; de hecho, las tasas de actividad de los municipios de Padrón y de Nigrán son altas, una vez corregidos los datos del padrón que subregistraban la agricultura. En general, la TAF elevada del mundo 
rural obedecía a que en Galicia predominaba la pluriactividad y a que las familias trabajaban en una agricultura minifundista sumando esfuerzos y alternando las tareas del campo con las textiles y el curtido (Padrón), las de la pesca-conserva (Bueu) o las agrario-pesqueras (la parroquia de Panxón en Nigrán).

También influían positivamente en la participación laboral femenina algunos factores de oferta. En primer lugar, la edad, constatándose que en el rural gallego las mujeres trabajan hasta edades avanzadas. En segundo lugar, el estado civil, que apenas tenía influencia en el rural, con TAF elevada para solteras, casadas y viudas, pero sí en las ciudades, donde las solteras eran las que presentaban una mayor ocupación, trabajando principalmente de criadas, tal y como acontecía en el siglo xIX, ocupación que solía abandonarse al acceder al matrimonio. En tercer lugar, la actividad de las casadas no se detenía con la maternidad, sino que la TAF se mantenía relativamente estable independientemente del número de hijos. Por último, el salario del marido influía en la actividad de las casadas, de forma que, si este no alcanzaba para sostener la economía familiar, las mujeres trabajaban fuera del hogar, de modo que no se aplica el modelo de ganador de pan a la Galicia de la década de 1920. Estos resultados todavía serían más robustos si se hubiera podido corregir el subregistro en todas las actividades económicas y no sólo en la agricultura, en la pesca y su transformación.

\section{AGRADECIMIENTOS}

Esta investigación ha sido financiada por el proyecto «La estructura de la ocupación y el ingreso en el largo plazo. Redefiniendo la modernización económica y los niveles de vida en Galicia, 1750-1975» (HAR2017-85601-C2-2-P). IP: Luisa Muñoz Abeledo. Agradecemos a los editores y evaluadores anónimos a quienes Historia Agraria encomendó la revisión de este artículo sus comentarios que, sin duda, han contribuido a mejorar la primera versión de este artículo. A María José Ginzo, del Departamento de Estadística e Investigación Operativa de la Universidad de Santiago de Compostela, su colaboración en el tratamiento estadístico de los datos de actividad.

\section{REFERENCIAS}

Ansola, A. (1996). Cambio económico y modo de vida en las comunidades cántabras (siglos XIX Y XX). Tesis doctoral. Santander: Universidad de Cantabria.

Arbaiza, M. (2000). La "cuestión social» como cuestión de género: Feminidad y trabajo en España, 1860-1930. Historia Contemporánea, (21), 395-458. 
ARbaiza, M. (2002). La construcción social del empleo femenino en España (1850-1935). Arenal: Revista de historia de las mujeres, 9 (2), 215-239.

ARBAIZA, M. (2003). Orígenes culturales de la división sexual del trabajo en España (18001935). En C. Sarasúa \& L. Gálvez MuÑoz (Coords.), ¿Privilegios o eficiencia?: Mujeres y hombres en los mercados de trabajo (pp. 189-217). Alicante: Universidad de Alicante. ARTAZA, R. DE (1959). La villa de Muros y su distrito. Pontevedra: Ayuntamiento de Muros.

Ballesteros, E. (2002). Contribuciones de las mujeres al bienestar material de los hogares en la España contemporánea: Aproximaciones socio-históricas. Arenal: Revista de historia de las mujeres, 9 (2), 241-267.

BLANCO, F. (2001). Los cambios sociales en A Coruña durante el primer tercio del siglo Xx. En X. BALbOa \& H. Pernas (Coords.), Entre nós: Estudios de arte, xeografía e historia en homenaxe ó profesor Xosé Manuel Pose Antelo (pp. 1245-1262). Santiago de Compostela: Universidade de Santiago de Compostela.

Borderías, C. (1993). Entre líneas de trabajo e identidad femenina en la España contemporánea. Barcelona: Icaria.

Borderías, C. (2002). El trabajo de las mujeres en la Cataluña contemporánea desde la perspectiva de los hogares: Balance y perspectivas. Arenal: Revista de historia de las mujeres, 9 (2), 269-300.

Borderías, C. (2003). La transición de la actividad femenina en el mercado de trabajo barcelonés (1856-1930):Teoría social y realidad histórica en el sistema estadístico moderno. En C. SARAsÚa \& L. Gálvez MuÑoz (Coords.), ¿Privilegios o eficiencia?: Mujeres y hombres en los mercados de trabajo (pp. 241-276). Alicante: Universidad de Alicante.

BORDERÍAS, C. (2010a). La reconstrucción de la tasa de actividad en la Cataluña industrial: Nuevas evidencias sobre los determinantes del empleo femenino (siglos XIX-XX). IX Congreso de la Asociación de Demografía Histórica, Sao Miguel, Açores. Universidade das Açores.

BorderíAs, C. (2010b). Revisiting Female Activity Rates in $19^{\text {th }}$ Century Catalonia. ESF Exploratory Workshop, «Reconstructing the Female Labour Force Participation Rates in Western Europe, $18^{\text {th }}$ and $19^{\text {th }}$ centuries». Barcelona, 4-6 de noviembre.

BORDERÍAS, C. (2012). La reconstrucción de la actividad femenina en Cataluña circa 1920. Historia Contemporánea, (44), 17-47.

BorderíAs, C. (2013a). Revisiting Women's Labor Force Participation in Catalonia's Textil Industry, 1920-1936. Feminist Economics, 19 (4), 224-242.

Borderías, C. (2013b). Salarios infantiles y presupuestos familiares en la Cataluña Obrera, 1856-1920. En J. M. BorRÁs (Coord.), El trabajo infantil en España (17001950) (pp. 371-408). Barcelona: Icaria.

Borderías, C. \& FERrer, L. (2015). Hogar e industria textil: Mercado de trabajo y estrategias familiares en Catalunya (1900-1936). Historia Social, (81), 3-27. 
Borderías, C. \& FERRER, L. (2017). The Stem Family and Industrialization in Catalonia (1900-1936). The History of the Family, (22), 34-56.

Borderías, C. \& López Guallar, P. (2003). A Gendered View of Family Budgets Midnineteenth Century Barcelona. Histoire et mésure, (18), 113-146.

BorderíAs, C. \& MuÑoz ABELEDO, L. (2018). ¿Quién llevaba el pan a casa en la España de 1924?:Trabajo y economías familiares de jornaleros y pescadores en Cataluña y Galicia. Revista de Historia Industrial, (74), 77-106.

BorRÁs, J. M. (2012). Tasas de actividad infantil y género en la Cataluña de 1900: Estudio de casos de la cuenca del Ter. Historia Contemporánea, (44), 73-108.

BorRÁs, J. M. (Ed.) (2013). El trabajo infantil en España (1700-1950). Barcelona: Icaria.

Campos, C. (2014). Female Labour Force Participation Rates in the 19th Century Andalusia: Antequera. Investigaciones de Historia Económica, 10 (3), 191-201.

CAMPS, E. (1991). Els nivells de benestar al final del segle XIx: Ingrés i cicle de formació de les famílies a Sabadell, 1890. Recerques, (24), 7-21.

CAMPs, E. (1995). La formación del mercado de trabajo industrial en la Cataluña del siglo XIX. Madrid: Ministerio de Trabajo y Seguridad Social.

CAMPS, E. (1999). De ocupación, sus labores: El trabajo de la mujer en los albores del siglo Xx (Sabadell, 1919-1920). En K. ZegarRa \& M. GonZÁlez Portilla (Coords.), IV Congreso de la Asociación de Demografía Histórica (pp. 549-562). Vol. 2. Bilbao: Universidad del País Vasco.

Carmona, J. (1997). O mar e a industrialización de Galicia. En G. Pereira-Menaut (Coord.), Galicia fai dous mil anos: O feito diferencial galego (pp. 251-276).Vol. 2. Santiago de Compostela: Museo do Pobo Galego.

Carmona, X. \& NADAL, J. (2005). El empeño industrial de Galicia: 250 años de historia (1750-2000). A Coruña: Fundación Pedro Barrié de la Maza.

Cooper, C. (1992). Native Women of the Northern Pacific Coast: An Historical Perspective, 1830-1900. Fournal of Canadian Studies, 27 (4), 44-75.

EsCUDERO, A. (2002).Volviendo a un viejo debate: El nivel de vida de la clase obrera británica durante la Revolución industrial. Revista de Historia Industrial, (21), 13-62. EsCUDERO, A. (2003). El bienestar en España: Una perspectiva de largo plazo, 1850-1991. Revista de Historia Económica/fournal of Iberian and Latin American Economic History, 21 (3), 525-565.

Escudero, A. \& Pérez Castroviejo, P. (2010). The Living Standard of Miners in Biscay (1876-1936): Wages, the Human Development Index and Height. Revista de Historia Económica/fournal of Iberian and Latin American Economic History, 28 (3), 503534.

FolbRE, N. (1995). The Unproductive Housewife: Her Evolution in Nineteenth Century Economic Thought. En J. HumphriEs (Ed.), Gender and Economics (pp. 217-234). Aldershot: Edward Elgar. 
FREIRE, M. P. (2008). A familia rural na Galicia contemporánea: O cambio nas relacións familiares nos concellos de Brión e Padrón (1850-1970). Santiago de Compostela: Lóstrego.

GÁlvez MuÑoz, L. (1997). Breadwinning Patterns and Family Exogenous Factors:Workers at the Tobacco Factory of Seville during the Industrialization Process, 1887-1945. International Review for Social History, 42 (5), 87-129.

GÁlvez MuÑoz, L. (2000). Compañia arrendataria de tabacos (1887-1945): Cambio tecnológico y empleo femenino. Madrid: LID.

GARRIDO, L. (2016). La tasa de actividad femenina en el siglo XVIII en dos municipios andaluces: Laujar de Andarax (Almería) y Úbeda (Jaén). Investigaciones de Historia Económica, 12 (3), 144-153.

GIRÁlDEZ, J. (1996). Crecimiento y transformación del sector pesquero gallego, 18801936. Madrid: Ministerio de Agricultura, Pesca y Alimentación.

GonzÁlez Portilla, M. (Ed.) (2009). La consolidación de la metrópoli de la Ría de Bilbao. 1: Segunda industrialización, inmigración y capital humano. Bilbao: Fundación BBVA. Grantham, G. (2012). Occupational, Marital, and Life-Cycle Determinants of Women's Labor Force Participation in Mid Nineteenth-Century Rural France. Feminist Economics, 18 (4), 97-119.

Grantham, G. \& GRIMARD, F. (2010). The Industrious Revolution and Labour Force Participation of Rural Women: Evidence from Mid-Ninetenth Century France. En L. CRUZ \& J. MOKYR (Eds.), The Birth of Modern Europe: Culture and Economy, 14001800: Essays in Honor of Fan de Vries (pp. 187-214). Leiden: Brill. (Library of Economic History, 2).

Hastie, T. \& Tibshirani, R. (1990). Generalized Additive Model. London/New York: Chapman and Hall.

Hernández García, R. \& Cubero, J. (2017). La Tierra de Campos de Valladolid a mediados del siglo XVIII: Estudio y transcripción de las respuestas generales del Catastro de la Ensenada.Valladolid: Diputación Provincial de Valladolid.

HigGs, E. (1987). Women, Occupations and Work in the Nineteenth Century Censuses. History Workshop Fournal, 23 (1), 59-80.

HigGS, E. (1995). Occupational Censuses and the Agricultural Workforce in Victorian England and Wales. The Economic History Review, 48 (4), 700-716.

Higgs, E. (2005). Making Sense of the Census Revisited: Census Records for England and Wales, 1801-1901. London: Institute of Historical Research.

Homobono, J. I. (1993). Las conservas de pescado en el País Vasco. En J. I. Homobono (Ed.), Conservas de pescado y litografía en el litoral Cantábrico (pp. 11-61). Madrid: FEVE.

HoRRELL, S. \& HUMPHRIES, J. (1995). Women's Labour Force Participation and the Transition to the Male Breadwinner Family, 1790-1865. The Economic History Review, 48 (1), 89-117. 
Horrell, S. \& Humphries, J. (1997). The Origins and Expansion of the Male Breadwinner Family: The Case of Nineteenth-Century Britain. International Review of Social History, 42 (S5), 25-64.

Humphries, J. \& SARASÚA, C. (2012). Off the Record: Reconstructing Women' Labor Force Participation in the European Past. Feminist Economics, 18 (4), 39-68.

Janssens, A. (1997). The Rise and Decline of the Male Breadwinner Family?: An Overview of the Debate. International Review of Social History, 42 (S5), 1-23.

Jover, G., Pujadas, J. M. \& SuAU, A. M. (2017). ¿Quiénes eran los mozos en las regiones mediterráneas?: Salarios y movilidad de los mozos en el mercado de trabajo de Mallorca, 1654-1680. Mundo Agrario, 18 (39).

LANA, J. M. (2007). El poder de compra de jornaleros y criados: Salarios reales y mercados de trabajo en la Navarra rural (1781-1936). Investigaciones de historia económica, (7), 37-68.

LINDOSO, E. \& MiRÁs, J. (2001). La trayectoria de una economía urbana: A Coruña (18681936). En E. Grandío, A. Romero \& X. Alfeirán (Eds.), El Republicanismo coruñés en la historia (pp. 31-38). A Coruña: Ayuntamiento de A Coruña.

LLONCH, M. (2004). Jornada, salarios y costes laborales en el sector textil catalán (18911936). Revista de historia industrial, (26), 101-140.

Maluquer de Motes, J. (2005). Consumo y precios. En A. Carreras, C. Barciela \& X. TAFUNELL (Coords.), Estadísticas históricas de España, siglos XIX-XX (1247-1296). Bilbao: Fundación BBVA.

MALUQUER DE Motes, J. (2006). «La paradisiaca estabilidad de la anteguerra»: Elaboración de un índice de precios de consumo en España, 1830-1936. Revista de Historia Económica/fournal of Iberian and Latin American Economic History, 24 (2), 333-382.

MALUQUeR DE Motes, J. (2013). La inflación en España: Un índice de precios de consumo, 1830-2012. Estudios de historia económica, (64), 1-147.

MARTíNeZ López, D. \& MARTÍNEZ MARTíN, M. (2003). El trabajo femenino y la economía campesina de subsistencia en Andalucía: Las hilanderas de Montefrío (1826-1851). En C. Sarasúa \& L. Gálvez MuÑoz (Coords.), ¿Privilegios o eficiencia?: Mujeres y hombres en los mercados de trabajo (pp. 137-156). Alicante: Universidad de Alicante.

Martínez Martín, M. \& Moya, G. (2011). Trabajo y actividad en la configuración de la ciudad andaluza de Granada entre 1890 y 1930. En A. PAREJA, El capital humano en el mundo urbano: Experiencias desde los padrones municipales (1850-1930) (pp. 127144). Bilbao: Universidad del País Vasco.

MARTínez Soto, A. (2013). La construcción de la identidad política y sindical de las jornaleras del campo en la zona vitivinícola del sureste de España, 1900-1936. XIV Congreso Internacional de Historia Agraria. Badajoz, 7-9 de noviembre.

MIRÁs, J. (2004). Caracterización del sector comercial en A Coruña durante el primer tercio del siglo xx. Perspectivas Urbanas, (4), 1-9. 
MuÑoz Abeledo, L. (2002). Los mercados de trabajo en las industrias maritimas de Galicia: Una perspectiva histórica, 1870-1936. Tesis doctoral. Barcelona: Universidad Autónoma de Barcelona.

MuÑoz Abeledo, L. (2010). Género, trabajo y niveles de vida en la industria conservera de Galicia (1870-1970). Barcelona: Icaria.

MuÑoz ABELEDo, L. (2012a). Actividad femenina en industrias pesqueras de España y Portugal (1870-1930). Historia Contemporánea, (44), 49-72.

MuÑoz Abeledo, L. (2012b). Women in the Rural and Industrial Labor Force in Nineteenth Century Spain. Feminist Economics, 18 (4), 121-143.

MuÑoz Abeledo, L. (2013). El trabajo infantil en las industrias pesqueras de España, 1850-1936. En J. M. BorRas (Coord.), El trabajo infantil en España (1750-1950) (pp. 117-152). Barcelona: Icaria.

MuÑz Abeledo, L., TABoAdA, S. \& Verdugo, R. (2015). Condicionantes de la actividad femenina en la Galicia de mediados del siglo xIx. Revista de Historia Industrial, (59), 39-80.

Nederveen MeERKerk, E. van (2010). National Registrations, Additional Sources and Female Labor Force Participation Rates: The Netherlands in the $19^{\text {th }}$ Century. European Science Foundation Exploratory Workshop, «Reconstructing the Female Labor Force Participation Rates in Western Europe, $18^{\text {th }}$ and $19^{\text {th }}$ centuries». Barcelona, noviembre.

ORTEGA, T. M. (2015). Fornaleras, campesinas y agricultoras: La historia agraria desde una perspectiva de género. Zaragoza: Prensas de la Universidad de Zaragoza.

Otero, R. (1973). Orense. Vigo: Bibliófilos gallegos.

Pérez Castroviejo, P. (1992). Clase obrera y niveles de vida en las primeras fases de la industrialización vizcaína. Madrid: Ministerio de Trabajo y Seguridad Social.

Pérez Castroviejo, P. (2006). Poder adquisitivo y niveles de vida de los trabajadores vizcaínos. Revista de Historia Industrial, 15 (30), 103-141.

PÉREZ-FueNTES, P. (1993). Vivir y morir en las minas: Estrategias familiares y relaciones de género en la primera industrialización vizcaína (1877-1913). Bilbao: Universidad del País Vasco.

PÉrez-Fuentes, P. (1995). El trabajo de las mujeres en España en los siglos XIX y Xx: Consideraciones metodológicas. Arenal. Revista de historia de las mujeres, 2 (2), 219-245.

PÉrez-Fuentes, P. (2003). «Ganadores de pan» y «amas de casa»: Otra mirada sobre la industrialización vasca. Bilbao: Universidad del País Vasco.

Pérez-Fuentes, P. (2013). Women's Economic Participation on the Eve of Industrialization: Bizkaia, Spain, 1825. Feminist Economics, 19 (4), 160-180.

Pérez-Fuentes, P. \& Pareja, A. (2011). La evolución de las tasas de actividad femenina en Vizcaya (1825 1935) a través de los padrones de población. X Congreso In- 
ternacional de la Asociación Española de Historia Económica. Carmona, 8-9 de septiembre.

Pérez-Fuentes, P., Pareja, A. \& Zarraga, K. (2010). Actividad femenina y estrategias de los hogares en el País Vasco: Una visión comparada en los años 20. IX Congreso de la Asociación de Demografía Histórica, Sao Miguel, Açores. Universidade das Açores.

Pettersen, L. T. (1996). Crisis Management and Household Strategies in Lofoten: A Question of Sustainable Development. Sociología Ruralis, 36 (2), 237-248.

Precedo, A. J. (1990). La Coruña, metrópoli regional. A Coruña: Fundación Caixa Galicia.

SÁnchez Cidrás, A., Cerviño, X. \& Fernández Aldegunde, M. (1998). A industria da pesca salgada: Os portos de Bueu e Beluso. Santiago de Compostela: Xunta de Galicia, Dirección Xeral de Formación Pesquería e Investigación.

SARASÚA, C. (2000). El análisis histórico del trabajo agrario: Cuestiones recientes. Historia Agraria, (22), 79-96.

SARAsÚA, C. (2013). ¿Activos desde cuándo?: La edad de acceso al mercado de trabajo en la España del siglo XviII. En J. M. BorRÁs (Coord.), El trabajo infantil en España (1750-1950) (pp. 61-80). Barcelona: Icaria.

SARASÚA, C. (2019). Women's Work and Structural Change: Occupational Structure in Eighteenth-Century Spain. The Economic History Review, 72 (2), 481-509.

SiLvestre, J. (2005). El grado de discriminación salarial de las mujeres en España, 1930: Una primera aproximación. Investigaciones de historia económica: revista de la Asociación Española de Historia Económica, (2), 105-144.

Somoza, J. (2000). Dinámica industrial de Ourense en el siglo xx. Polígonos, (10), 117 136.

Somoza, J. (2001). Desarrollo urbano en Ourense, 1895-2000. Tesis doctoral. Santiago de Compostela: Universidade de Santiago de Compostela.

VAlCÁrCel, M. (1999). Ourense, a grande cidade literaria. En C. Díaz MartíneZ, M. Valcárcel \& X. C. Caneiro, A memoria de Ourense: Album de postais (pp. 86-89). Vigo: Xerais de Galicia.

Verdon, N. (2002). Rural Women Workers in Nineteenth Century England: Gender, Work and Wages. Woodbridge: Boydell Press.

VILAR, M. (2014). Los diferenciales salariales entre mujeres y hombres en España (c. 1850-1975): Un análisis provisional. Áreas. Revista internacional de ciencias sociales, (33), 63-85.

VILLARES, R. (1982). La propiedad de la tierra en Galicia, 1500-1936. México: Siglo XXI. Wood, S. N. (2017). Generalized Additive Models:An Introduction with R. 2nd ed. Boca Raton: Chapman \& Hall/CRC Press. 